IAU Colloquium 164: Radio Emission from Galactic and Extragalactic Compact Sources

ASP Conference Series, Vol. 144, 1998

J. A. Zensus, G. B. Taylor, E J. M. Wrobel (eds.)

\title{
A MERLIN and VLBI Survey of Faint Compact Radio Sources
}

\author{
M. A. Garrett \\ JIVE, Postbus 2, 7990 AA, Dwingeloo, The Netherlands
}

S. T. Garrington

U. of Manchester, NRAL, Jodrell Bank, Macclesfield, Cheshire, SK11 9DL, U.K.

\begin{abstract}
We have selected a field from the VLA FIRST Survey which is typical in all aspects except one: it contains a bright, but extremely compact VLBI calibrator $-\mathrm{J} 1159+291$. 127 unresolved FIRST sources with $S_{\text {FIRST }} \geq 10 \mathrm{mJy}$ lie within $2.5^{\circ}$ of this calibrator. $\lambda 6 \mathrm{~cm}$ MERLIN observations with a resolution $\sim 60$ mas detect around half the sources. These detections form the basis of a sample of faint but compact radio sources which are ideally suited to follow-up VLBI observations.
\end{abstract}

\section{The Faint Source Sample and MERLIN Observations}

Present day, large-scale VLBI surveys have so far focussed on samples of relatively bright (and often flat spectrum) radio sources with total flux densities typically > $350 \mathrm{mJy}$. By adopting these selection criteria, VLBI surveys have been remarkably successful, but by only targeting the very brightest objects, our current knowledge and general understanding of compact radio sources is surely incomplete.

The technique of phase-referencing now allows sources as faint as $1 \mathrm{mJy}$ per beam to be detected and reliably imaged. We have recently embarked on a faint source VLBI survey, which employs a novel observing strategy. We have defined a sample of 127 sources which were drawn from the VLA FIRST survey (Becker, White, \& Helfand 1995) and these satisfy the following simple selection criteria, all sources: (i) lie within a "selection box" $3^{\circ} \times 4^{\circ}$ wide, centered on the VLBI calibrator J1159+291, (ii) have measured FIRST sizes $\leq 5$ arcsec and, (iii) have FIRST peak fluxes $>10 \mathrm{mJy} /$ beam.

The advantages of this approach are considerable: (i) a relatively unbiased source sample arises naturally, (ii) by focusing on one small field scheduling is effortless and observing efficiency maximized (the long telescope slew times usually associated with snapshot surveys are eliminated), (iii) measurement of source redshifts (using multi-fiber optical spectroscopy) requires only a modest amount of telescope time, (iv) by careful selection of a "model" calibrator, the data analysis can be largely automated and high SNR (delay, rate and phase) solutions can be obtained with very short calibrator integration times, (v) the offset on the sky between the source and the calibrator is typically much less than $2.5^{\circ}$, even for relatively large samples, and finally (vi) the inverse process of attempting to identify phase-calibrators for every target in a large faint source sample, is happily avoided.

Our 127 FIRST sources were observed by MERLIN at $\lambda 6 \mathrm{~cm}$ in January this year. Each source was observed in snapshot, phase-reference mode for a total of 15 minutes spread over $12 \mathrm{hr}$ in hour angle. The data were analyzed automatically. with the phase and amplitude corrections of the calibrator (J1159+291) being applied to each target source. The final maps had an r.m.s. noise of $0.3 \mathrm{mJy} /$ beam and a resolution of $60 \mathrm{mas}$ (see Fig. 1). Of the 127 sources we 
observed with MERLIN, 68 were clearly detected $(7 \sigma) .5$ of these were observed to be very extended and are most likely the lobes of extended radio sources which appear as "doubles" in the FIRST maps. The MERLIN detection rate is impressive, especially when one considers that the $\lambda 6 \mathrm{~cm}$ beam area is a factor of 10000 smaller than FIRST's.

Preliminary MERLIN $\lambda 18 \mathrm{~cm}$ images show that the majority of sources have little intermediate structure $(0.5-3.0$ arcsec $)$. Our high detection rate is interesting, the evolutionary models of Wall \& Jackson (1997) predict that the fraction of flat spectrum objects should decrease to $10-20 \%$ at these flux levels.
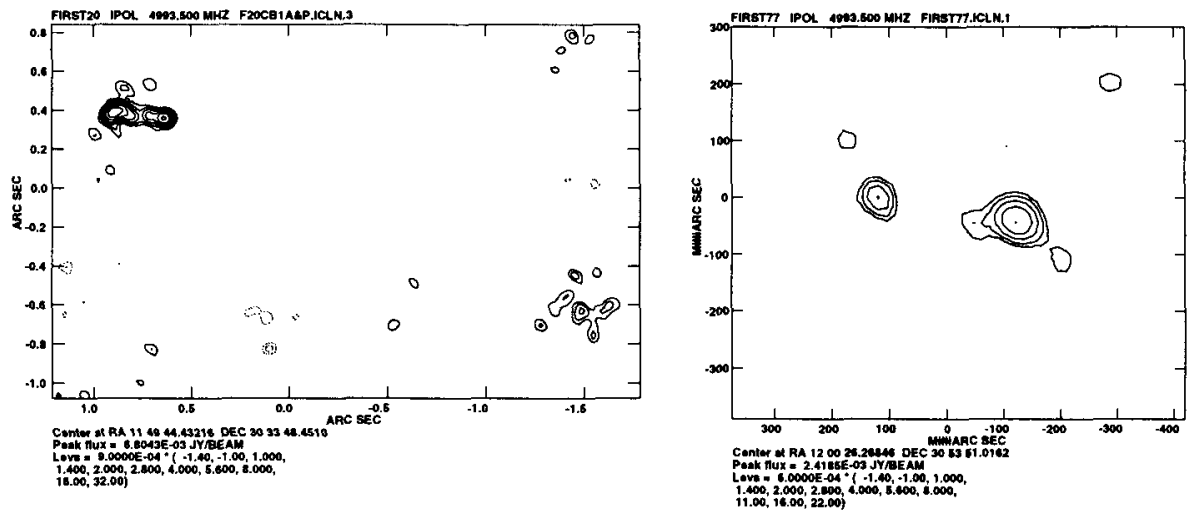

Figure 1. Two of the faint FIRST sources which were detected by MERLIN at $6 \mathrm{~cm}$.

\section{Motivation and Current Status}

For the first time, the combination of the FIRST survey, MERLIN filter observations and the phase-reference technique, allows us to reliably image and systematically classify a large number of faint, compact radio sources which are 1 to 2 orders of magnitude fainter than those targeted in previous VLBI surveys. We intend to compare the properties of these faint sources with their brighter cousins. By going deeper we hope to uncover a few surprises too. Phase-reference MERLIN-VLBI observations have already begun at $\lambda 18 \mathrm{~cm}$ and further $\lambda 6 \mathrm{~cm}$ VLBI and MERLIN observations are planned. In the spirit of the FIRST project the MERLIN $\lambda \lambda 6$ and $18 \mathrm{~cm}$ images and further information are available on-line at: http://www.nfra.nl//mag/first.html.

\section{References}

Becker, R. H., White, R. L., \& Helfand, D. J. 1995, ApJ, 450, 559-577.

Wall, J. V., \& Jackson, C. A. 1997, MNRAS, in press. 